\title{
Professionelle Karriereförderung auf dem Weg zur Professorin oder Chefärztin
}

Regine Landmann, Anne von Gunten

Korrespondenzen: Prof. Dr. Regine Landmann Div. Infectious Diseases Department of Research University Hospital

Hebelstrasse 20

CH-4031 Basel

Tel. 0612652325 office

Tel. 0612652366 laboratory

Fax 0612652350

regine.landmann@unibas.ch

Anne von Gunten, lic. phil. Department of Research

University Hospital

Hebelstrasse 20

CH-4031 Basel

Tel. 0313092350

anne.vongunten@unibas.ch

\section{Hintergrund}

Der Bund hatte sich 2000 für die Durchführung eines Programms Chancengleichheit entschieden, weil 1998 nur 7\% der Professuren an schweizerischen Universitäten von Frauen besetzt waren [1]. Die Medizin weist zwar auf der Ebene der Studierenden einen deutlich höheren Frauenanteil auf als andere naturwissenschaftlichen Fachbereiche in der Schweiz, allerdings sinkt dieser Anteil mit steigender Karrierestufe stark ab: Im Jahr 2005 schlossen 48,6\% Frauen ihr Studium und 47,2\% Frauen ihr Doktorat an der Medizinischen Fakultät der Universität Basel ab [2], während im selben Jahr nur 10,1\% Frauen als Professorinnen tätig waren (Abb. 1).

Die Geschlechterverhältnisse auf Ebene der Professuren an den Schweizer Medizinfakultäten sind in Abbildung 1 dargestellt und zeigen ein stark unausgewogenes Bild. Der Frauenanteil aller Medizinfakultäten lag 2004 zwischen 7,9 und $10,1 \%$ (Abb. 1). Soll das für die BTIBotschaft 2008-2011 vorgeschlagene Ziel bis 2011 erreicht werden und 1/4 aller Professuren mit Frauen besetzt sein, besteht für alle Schweizer Medizinfakultäten Handlungsbedarf.

Die Gleichstellungskommission der Medizinischen Fakultät begründete Massnahmen zur Erhöhung der Professorinnenanzahl mit zwei Argumenten:

1. Die Geschlechterverhältnisse auf Ebene der Professuren entsprechen nicht dem in der Schweiz demokratisch und verfassungsmässig festgelegten Grundsatz der Gleichstellung.

2. Neben ethischen bzw. demokratischen Argumenten greifen auch ökonomische Überlegungen: Der Schweiz gehen wichtige human resources verloren, wenn das vorhandene Potential unter den Nachwuchswissenschaftlerinnen nicht für die Konkurrenzfähigkeit der Schweiz im europäischen und interkontinentalen Kontext genutzt wird.

Die Untervertretenheit der Frauen ab einer bestimmten Hierarchiestufe (glass ceiling effect) in der Schweiz wird zum Teil den strukturellen Bedingungen zugeschrieben. Die fehlenden Teil-

\section{Promotion professionnelle sur la voie du professorat ou du médecin-chef}

En 2004 et 2005 un programme de mentorat pour les femmes assistants et chefs de clinique a été mené à la Faculté de médecine de Bâle. La présidente de la commission d'égalité à la faculté de médecine, le Prof. R. Landmann, a réalisé ce programme grâce aux moyens octroyés par le programme fédéral «Egalité des chances» (20042007). L'objectif était de promouvoir la relève en médecine, de soutenir les femmes dans leur plan de carrière ainsi que leur nomination en tant que professeur ou médecin-chef. Les mentors ont accompagné 25 femmes médecins pendant une année et organiser des ateliers qui ont servi aussi bien de réseaux d'information que d'échanges. Le programme s'est révélé un succès pour tous les inscrits. Les résultats de cette évaluation sont présentés dans la rubrique ci-après.

zeitstellen auf höheren Positionen und die fehlende, familienexterne, flexible Kleinkinderbetreuung werden angeführt.

Es stehen den jungen Ärztinnen aber auch eigene psychische Dispositionen und Verhaltensmuster im Weg. Subtile geschlechtsspezifische Wahrnehmungs- und Kommunikationsmechanismen entscheiden den Verlauf einer akademischen Karriere massgebend mit. So bestehen beispielsweise geschlechtsspezifisch unterschiedliche Tendenzen in der Selbstdarstellung (impression management) in Präsentationssituationen. Auch wird die wissenschaftliche Leistung von Frauen und Männern von beiden Geschlechtern systematisch verzerrt wahrgenommen, und es bestehen unterschiedliche Er- 
klärungsmuster für beruflichen Erfolg [3]. Diese Beobachtungen müssen für eine professionelle und geschlechtergerechte Nachwuchsförderung berücksichtigt werden.

Das Mentoringprogramm hatte somit ein doppeltes Ziel: Neben einer gezielten Laufbahnplanung sollten sowohl Mentorinnen und Mentoren wie auch Mentees auf die strukturell und sozialisatorisch bedingten Schwierigkeiten für Medizinerinnen auf der medizinischen Karriereleiter sensibilisiert werden.

\section{Ablauf der ersten Mentoringrunde am Universitätsspital Basel}

Allen 304 Assistenz- und 99 Oberärztinnen der Universitätskliniken Basel wurde eine Teilnahme am Mentoring für eine zielgerichtete Karriereförderung angeboten. Von den über 70 Bewerberinnen wurden 23 Medizinerinnen in das Programm aufgenommen, die sich bereits in oder kurz vor der Habilitationsphase befanden: Mit Ausnahme der 3 jüngsten Assistenzärztinnen hatten alle bereits mindestens einen FMH-Titel erworben, ihr Alter lag zwischen 29 und 45 Jahren und 14 dieser Frauen waren neben ihrem Engagement im Beruf auch Mutter.

Für alle am Programm teilnehmenden Medizinerinnen wurden fachnahe Mentorinnen oder Mentoren gesucht, die möglichst genau den
Wünschen der Mentees entsprachen. Die 23 Mentoringduos trafen sich während eines Jahres etwa alle 2-3 Monate, um konkrete Karrierefragen zu klären und berufliche Schritte zu planen.

Das Programmteam (Regine Landmann, Programmleitung; Anne von Gunten, Projektkoordinatorin; Catherine Müller, externe Beraterin; Beatrice Altorfer, Sekretariat) organisierte ausserdem drei Rahmenveranstaltungen mit Workshops zu Themen wie «Forschungsprojekte», «Work-Life-Balance», «Zeitmanagement» und «Führungspraxis» und unterstützte die Entscheidungs- und Zielfindungsprozesse innerhalb der Mentoringduos über anleitende Formulare, Vereinbarungen und Leitfäden.

\section{Resultate der Schlussevaluation}

\section{Ergebnisse der Mentees}

\section{Zielerreichung}

Das übergeordnete Ziel des Mentoringjahres war die Förderung von Medizinerinnen, die die Habilitation und/oder Chefärztinnenposition als Ziel angaben oder in Betracht zogen. Die individuelle Zielsetzung wurde von den Mentees in Absprache mit ihren Mentorinnen und Mentoren schriftlich festgelegt. Die Mentees verglichen ihre eigene, zu Beginn des Mentoringjahres abgegebene Zielformulierung mit ihren

\section{Abbildung 1}

Anzahl Professoren und Professorinnen der Schweizer Medizin- und Pharmaziefakultäten 2004 im Vergleich [4].

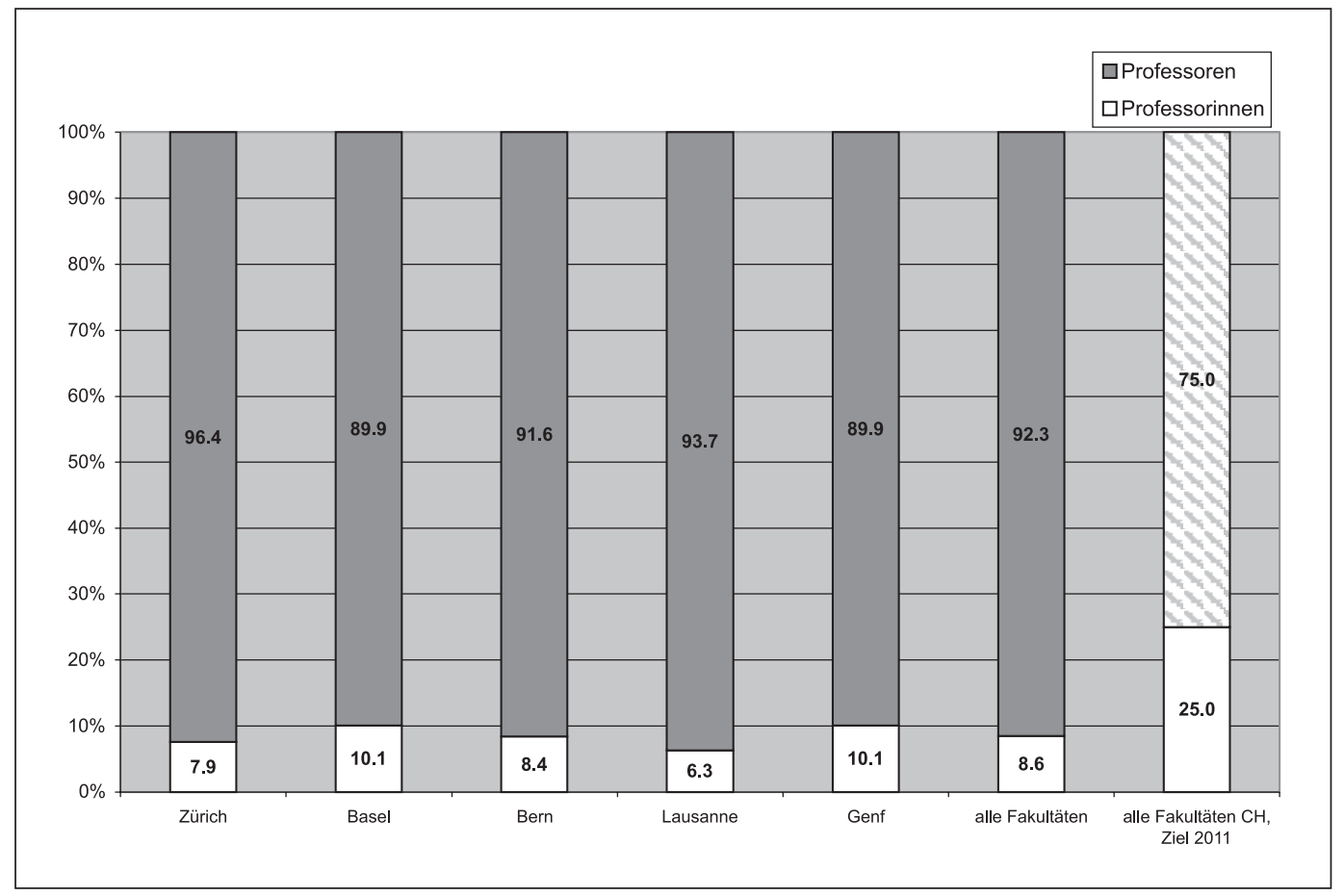


erreichten Zielen: 65\% der Mentees bewerteten ihre individuelle Zielsetzung als erreicht. Keine Mentee gab an, ihr Ziel gar nicht erreicht zu haben.

\section{Die beruflichen Schritte der Mentees} während des Mentoringjahres

Die konkret während des Mentoringjahres erreichten beruflichen Qualifikationen umfassten bei vier Mentees einen ersten FMH-Titel, bei vier weiteren Mentees einen zweiten FMH-Titel und bei einer Mentee die Habilitation.

Da der Weg zur Habilitation die Dauer des Programms überschreitet, wurden mit einem verfeinerten Raster auch die beruflichen Zwischenschritte erhoben. Auf Basis des Habilitationsreglements der Universität Basel gaben die Mentees an, welche der dort verlangten Schritte in Forschung, Lehre oder Arbeitsverhältnis sie während des Mentoringjahres realisieren, planen oder beginnen konnten (Abb. 3, Tab. 1).

Die Gewinne im Bereich der Forschung fielen insgesamt am stärksten ins Gewicht: Im Durchschnitt konnte jede Mentee im Verlaufe des Mentoringjahres fast drei Schritte realisieren. Fast jede Mentee nannte auch mindestens einen geplanten oder aktuellen Schritt. Nur wenige der geplanten oder begonnenen Schritte fielen in den Bereich des Arbeitsverhältnisses.

Die Evaluationsresultate lassen auf eine erfreuliche Zielstrebigkeit und konkrete Hand-
Tabelle 1

Zielvereinbarungen für die Zusammenarbeit zwischen Mentee und Mentorin oder Mentor.

\begin{tabular}{ll}
\hline Ziel & $\begin{array}{l}\text { Anzahl Nennungen } \\
\text { (1-3 Nennungen } \\
\text { pro Mentee) }\end{array}$ \\
\hline $\begin{array}{l}\text { Habilitation } \\
\begin{array}{l}\text { Beruflicher Aufstieg, } \\
\text { Erwerb von Führungs- } \\
\text { kompetenzen }\end{array}\end{array}$ & 10 \\
\hline $\begin{array}{l}\text { Etablierung im Spezialgebiet, } \\
\text { Festlegen eines Spezialgebietes }\end{array}$ & 9 \\
$\begin{array}{l}\text { Vereinbarkeit von Familie } \\
\text { und Beruf }\end{array}$ & 5 \\
$\begin{array}{l}\text { Beginn und Abschluss } \\
\text { von Forschungsprojekten }\end{array}$ & 5
\end{tabular}

lungsbereitschaft der Mentees schliessen. Diese Tatkraft äusserte sich in dem grossen Anteil der realisierten gegenüber geplanten oder laufenden beruflichen Schritten in Richtung Habilitation.

\section{Auswirkung des Mentorings auf die} subjektive Befindlichkeit der Mentees

- Der allgemeine Nutzen des Mentoringprogramms für die wissenschaftliche Karriere der Mentees wurde von 16 (70\%) als gross, von 7 (30\%) Mentees als «eher klein» beurteilt.

- $18(85,7 \%)$ Mentees waren insgesamt mit dem Mentoringprogramm zufrieden. Drei

\section{Abbildung 2}

Ablauf der ersten Mentoringrunde am Universitätsspital Basel 2004/2005.

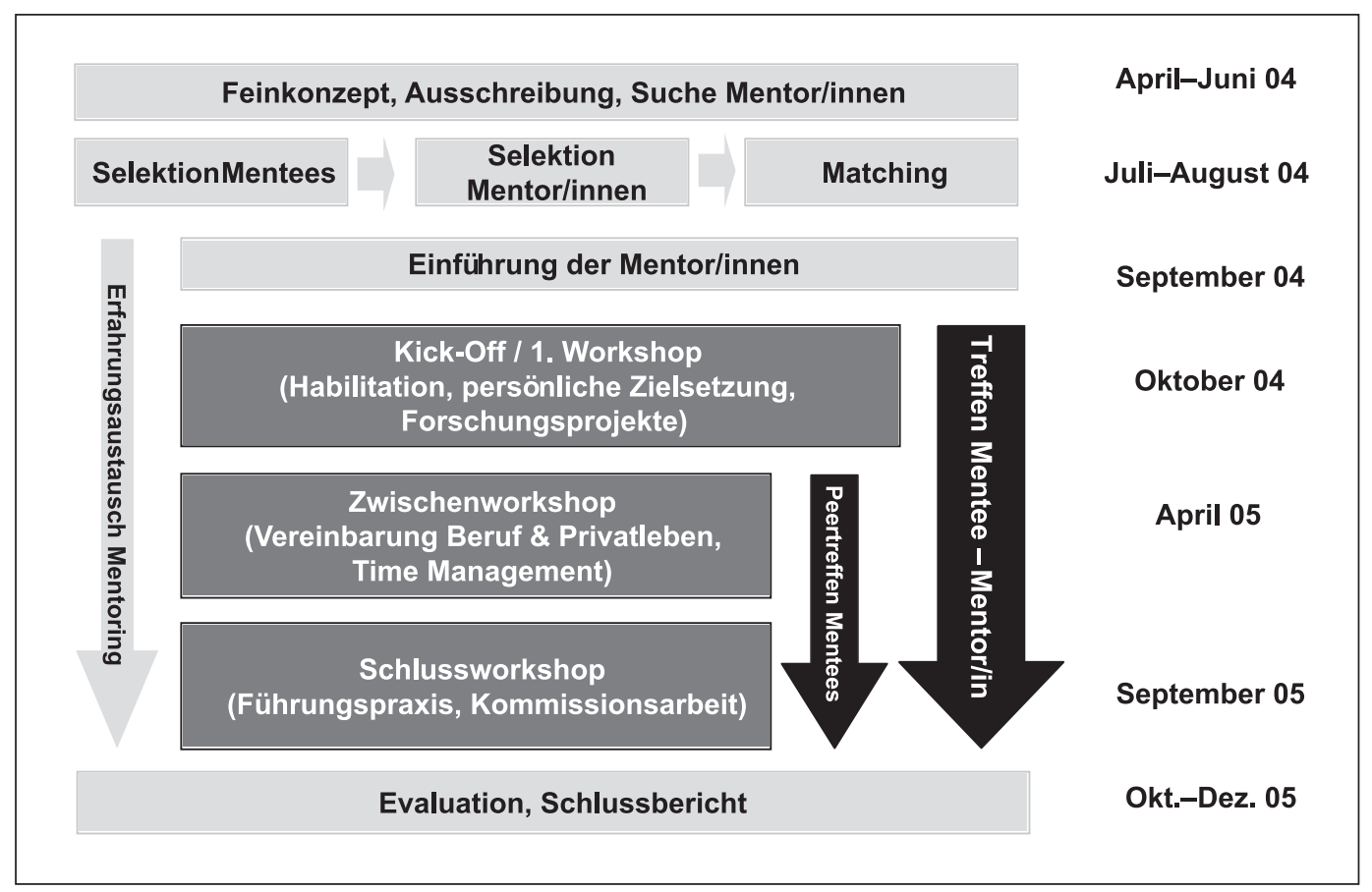


teilnehmende Medizinerinnen waren «eher unzufrieden», keine einzige Mentee gab ein negatives Feedback.

- 16 (69,5\%) Mentees wurden im Hinblick auf eine Habilitation positiv durch das Programm beeinflusst. Dabei wurden zwei Mentees auf die Idee gebracht und vierzehn in ihren Überlegungen, zu habilitieren, bestärkt. Drei Men- tees wurden durch die Einblicke, die sie in den Alltag ihrer Mentorinnen und Mentoren erhalten hatten, von dem Ziel der Habilitation abgebracht.

Für 20 Mentees hatte das Programm keine negativen Auswirkungen auf ihr berufliches Vorwärtskommen. Eine Medizinerin empfand eine

\section{Abbildung 3}

Schritte in Richtung Habilitation: Anzahl Nennungen in den Gebieten Forschung, Lehre, Arbeitsverhältnis ( $n=23$, Mehrfachnennungen).

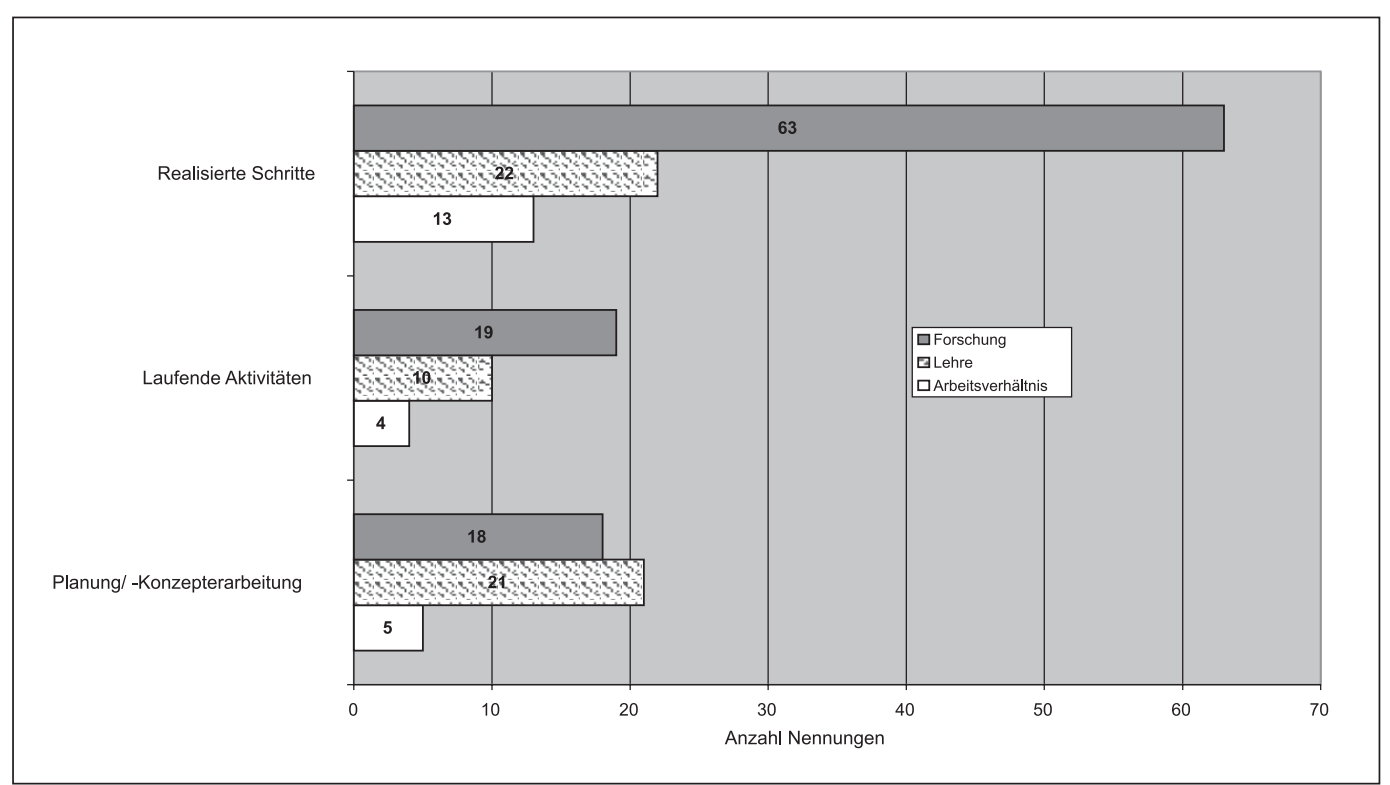

\section{Abbildung 4}

Einfluss der ersten Mentoringrunde auf die Haltung der Mentees zur Habilitation.

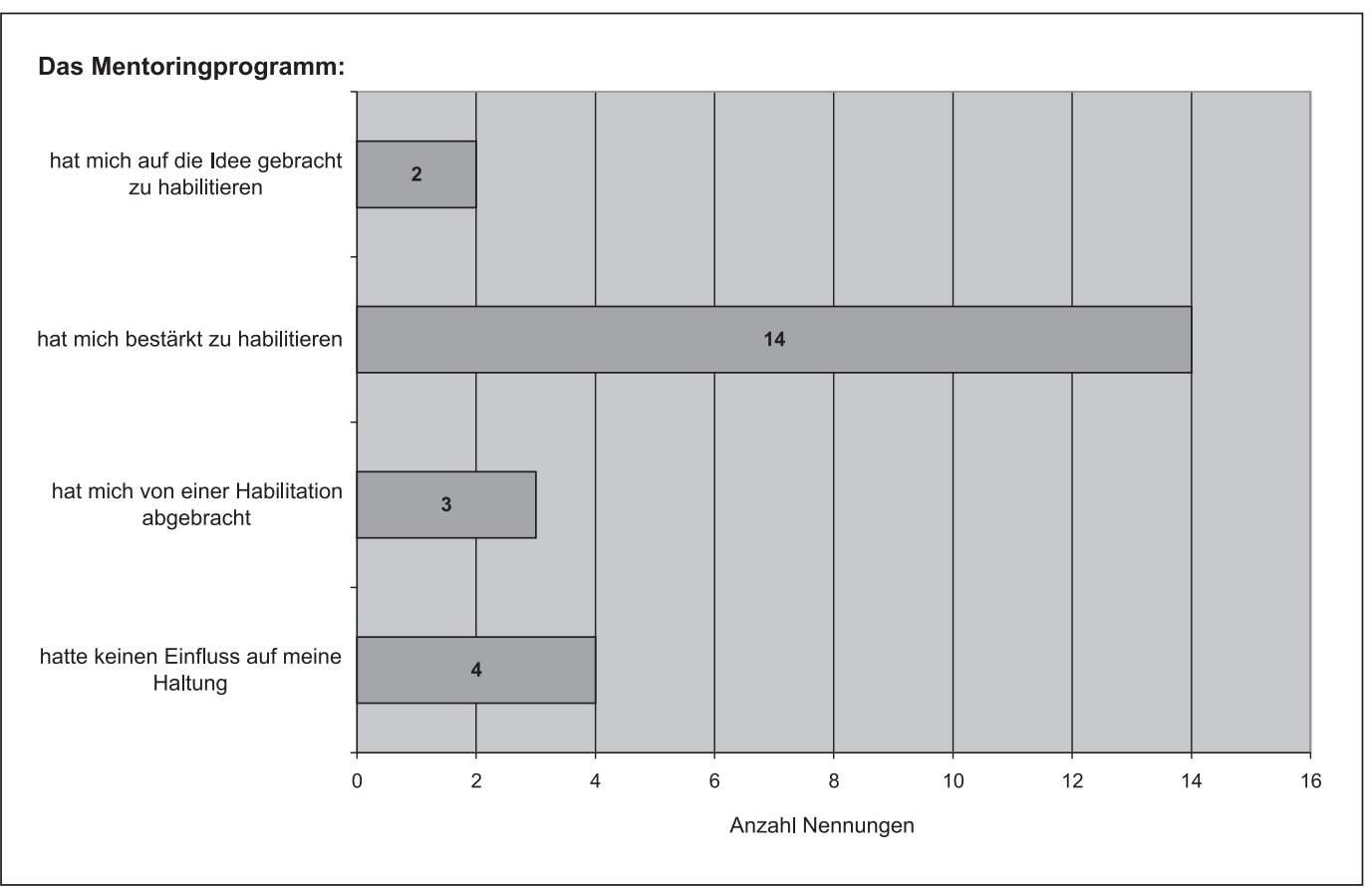


Abbildung 5

Gewinn der Mentorinnen und Mentoren im Mentoringprogramm 2004/2005.

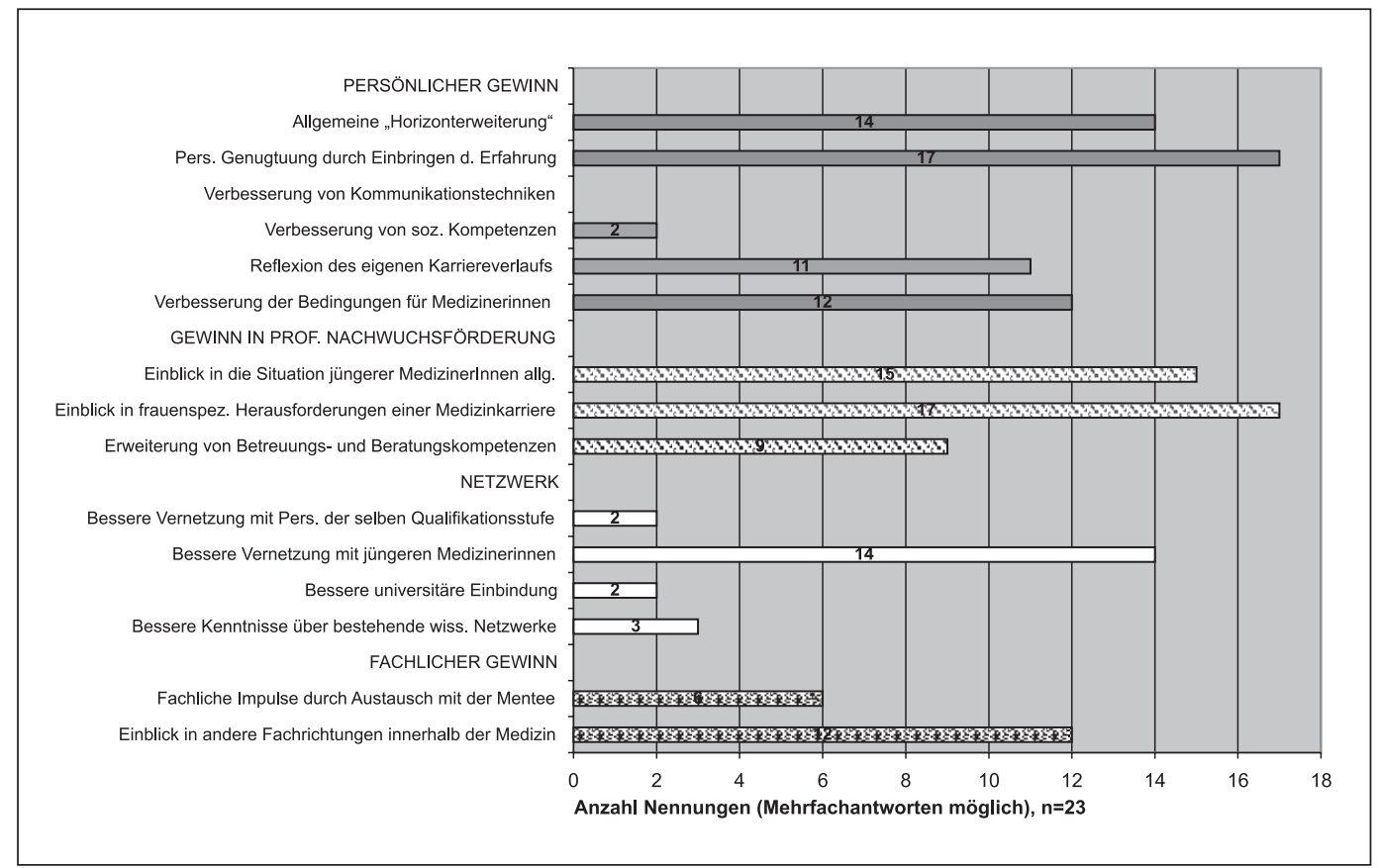

teilweise negative Auswirkung auf ihre Karriere durch die Teilnahme am Programm.

Weiterhin gaben 22 von 23 Mentees an, vom Einblick in die Karrierestrategien und den medizinischen Alltag der Mentorin oder des Mentors profitiert zu haben und mehr als $2 / 3$ fühlten sich beim zielgerichteten Arbeiten im Hinblick auf eine wissenschaftliche Karriere unterstützt. Ausserdem gaben 16 der 23 Mentees an, karrierewirksame Kenntnisse über wissenschaftliche Netzwerke gewonnen zu haben, und mehr als die Hälfte der Mentees wurden durch ihre Mentorin oder ihren Mentor im Hinblick auf ihre Forschungstätigkeit unterstützt.

\section{Ergebnisse Mentorinnen und Mentoren}

Die allgemeine Zufriedenheit der Mentorinnen und Mentoren mit dem Programm war gross: 20 von 23 Mentorinnen und Mentoren sprachen sich als zufrieden aus. Diese Haltung zeigte sich bei 21 von 23 Mentorinnen und Mentoren auch in der Bereitschaft, sich für weitere Mentoringrunden zur Verfügung zu stellen.

Die Mentorinnen und Mentoren beantworteten die Frage nach ihrem Gewinn durch ihre Teilnahme am Programm überwiegend positiv. 17 Mentorinnen und Mentoren erfuhren durch das Einbringen ihrer Erfahrung im Mentoringduo persönliche Genugtuung (Abb. 5). Ebenfalls 17 Mentorinnen und Mentoren erhielten durch das Mentoringprogramm Einblick in frauenspe- zifische Herausforderungen einer medizinischen Karriere (Abb. 5).

In diesem Ergebnis wurde ein Sensibilisierungseffekt des Programms sichtbar: 17 Professorinnen und Professoren wurden über persönliche Kontakte $\mathrm{zu}$ aufstrebenden, motivierten Medizinerinnen auf die Probleme der genderspezifischen Nachwuchsförderung aufmerksam gemacht. Interessant ist, dass 6 der 7 im Programm teilnehmenden Mentorinnen angaben, durch das Mentoring auf frauenspezifische Probleme sensibilisiert worden zu sein.

Interessanterweise gaben 12 der 23 Mentorinnen und Mentoren als Gewinn den Einblick in andere Fachrichtungen der Medizin ausserhalb ihres eigenen Spezialgebietes an.

Da die Mentorinnen und Mentoren aus ihrer Berufspraxis vermutlich bereits breite Kommunikations- und Sozialkompetenzen mitbrachten, waren in diesem Bereich keine Gewinne zu verzeichnen. Auch kamen die Mentorinnen und Mentoren über das Programm kaum miteinander in Kontakt, daher erstaunt auch der fehlende Gewinn zur Vernetzung über die Stufe der Mentees hinaus nicht (Abb. 5).

Es interessierte das Programmteam, ob und wie stark die Mentorinnen und Mentoren die Mentees a. in allgemeinen strategischen Karrierefragen; b. in fachspezifischen Karrierefragen; c. im psychosozialen Bereich; d. als Vorbild und e. beim Aufbau von Netzwerken unterstützten. 
In allen Bereichen gaben die Mentorinnen und Mentoren mehrheitlich an, für die Mentees «etwas» oder «grosse» Unterstützung geleistet zu haben. Am stärksten unterstützten die Mentorinnen und Mentoren (insgesamt 22 von 23) die Mentees in strategischen Karrierefragen.

\section{Zusammenfassung und Ausblick}

Sowohl auf Mentee- wie auch auf Mentorinnenund Mentorenseite zeigt sich der Erfolg des Programms: Der Prozess der Zielfindung und -erreichung der Mentees war zu einem hohen Grad erfolgreich und von etwa einem Viertel der Teilnehmerinnen konnten konkrete Qualifikationen erreicht werden. Die aktiven Karriereschritte der Mentees während des Mentoringjahres waren in ihrer Häufigkeit und Zielstrebigkeit erfreulich. Die Befragung zu den Befindlichkeiten auf Menteeseite zeigte, dass die zentralen Mentoringanliegen, wie beispielsweise die Steigerung der Karrieremotivation, erfüllt waren.

Auch die Mentorinnen und Mentoren sahen insgesamt das Mentoringjahr als Erfolg und zeigten sich in der Folge motiviert für die weitere Arbeit an einer professionellen und gendergerechten Nachwuchsförderung.

Von beiden Seiten her wird auf das grosse Engagement der Mentees hingewiesen, welches sich auch in dieser Durchführung als eine der Grundvoraussetzungen für ein erfolgreiches Mentoring erwiesen hat. Die Selektion der Mentees aufgrund ihrer Karriereorientiertheit hat sich somit bewährt. Es ist aber bekannt, dass sich Frauen im Hinblick auf ihr impression management in Reaktion auf die soziale Erwünschtheit tendenziell vorsichtiger als Männer verhalten. Vielfach werden von Frauen Karrierewünsche zurückhaltender geäussert, selbst wenn sie in ihren beruflichen Leistungen ihren männlichen Kollegen gleichstehen. Deshalb stellt sich die Frage, wie weit Mentoringprogramme, die das berufliche Selbstvertrauen von Frauen steigern sollen, in Selektionsphasen an der klar geäusserten Karrierebestrebung weiblicher Nachwuchskräfte orientiert sein dürfen.

Insgesamt bestätigte die Evaluation das Mentoring als geeignete Massnahme zur Nachwuchsförderung von Ärztinnen auf der Habilitationsstufe. Allerdings sollten die Ergebnisse der Schlussevaluation vorsichtig interpretiert werden, da keine Kontrollgruppe gebildet werden konnte. Für zukünftige Mentoringprogramme wird eine statistisch auswertbare Evaluation anhand von grösseren Kollektiven ins Auge gefasst.
Die mögliche Wirkung der ersten Mentoringrunde wird mit folgenden Überlegungen veranschaulicht: Im Jahr 2004 arbeiteten 10 Professorinnen und 89 Professoren an der Medizinischen Fakultät der Universität Basel. Würden alle 16 Frauen, die in unserer Evaluation angaben, durch die erste Programmrunde bestärkt oder auf die Idee gebracht worden zu sein, eine universitäre Karriere anzustreben, bis 2011 tatsächlich habilitieren und bei einer gleichbleibenden Anzahl Professuren an der Universität Basel ein Berufungsverfahren erfolgreich durchlaufen, wären bereits 26,3\% der Professuren an der Medizinischen Fakultät der Universität Basel von Frauen besetzt und das für die BTI-Botschaft 2008-2011 vorgeschlagene Ziel - ein Professorinnenanteil von $25 \%$ - wäre erreicht.

\section{Das Mentoringprogramm wird fortgesetzt ...}

Weil die Nachfrage nach Unterstützung mit über 70 Bewerberinnen sehr gross war und sich das angewandte Konzept bewährte, läuft seit Januar 2006 bereits die zweite Mentoringrunde am Department Forschung der Universitätskliniken Basel: 23 Assistenzärztinnen und Postdoktorandinnen haben zusammen mit ihren Mentorinnen und Mentoren die Arbeit im Duo aufgenommen und werden bis im Frühling 2007 vom Mentoringprogrammteam begleitet.

Um die Ziele einer professionellen und gendergerechten Nachwuchsförderung längerfristig verfolgen zu können, ist in den Augen des Mentoringprogrammteams die Institutionalisierung des Mentorings an Medizinischen Fakultäten erstrebenswert.

\section{Literatur}

1 Bachmann R, Rothmayr C, Spreyermann C. Evaluation Bundesprogramm Chancengleichheit von Frau und Mann an Universitäten. Bericht zu Umsetzung und Wirkungen des Programms 2000 bis 2003. Bern: Schriftenreihe Bundesamt für Bildung und Wissenschaft (BBW); 2004.

2 Nach Angaben des Medizinischen Dekanates der Universität Basel, 9. Mai 2006.

3 Baitsch C, Steiner E. Zwei tun das Gleiche. Kommunikation zwischen Frauen und Männern im Berufsalltag, Zürich: vdf Hochschulverlag AG. 2004

4 Jaberg U, Bencheikh M, Koller P. Personal der Universitären Hochschulen 2004. Bundesamt für Statistik (BFS). Neuchâtel: Statistik der Schweiz 2006. 